\title{
Practice makes perfect? Vets' information seeking behaviour and information use explored
}

Tim Wales

Department of Information Science, City University, Northampton Square, London, ECIV OHB tim@twales.freeserve.co.uk

A random sample of UK veterinary practitioners was surveyed and interviewed on behalf of the Royal College of Veterinary Surgeons Wellcome Library to identify key issues in veterinary information use (IU) and information seeking behaviour (ISB).' A greater proportion of respondents used the litemel for vetehary hom library. However, information sources used. Some variations in information source use by practice size and type and information type were identified. The majority of library users and nonusers wanted enhanced library access via the Internet, especially to full-text journals.

\section{Introduction}

sample of veterinarians in practice, to dis-

Librarianship and Information Science (LIS) : cover where and by what means they looked research in the field of veterinary science is : for the information they needed and what use rare - arguably, the last UK LIS study was car- : they made of it once found. It was intended ried out by a student at City University in 1993, : to be a localised, microenvironmental study, befored by the Internet This means that vet- : vironmental study on vets' information needs effectively operating in a vacuum with regard : to their users' needs, especially where elecronic information is concerned. Previous research

This has particular resonance for vets in : Pelzer and Leysen's 1991 survey of American practlce working 'on the front line', alone or - vets can be conn's pars intended (and funded) to support first and : established that books were the primary inforforemost the veterinary research undertaken - mation source used in critical care situations, by government or academic vets. Practising - and found that relatively little use was made vets who need help sourcing information will : of veterinary medical libraries, computer ultimately turn to the Royal College of : databases or extension services (2, 3]. The lat(RCVSWL), the 'profession's library' [1]. - Iy negative implications for vets' awareness of Accordingly, this research was undertak- : current issues in preventative medicine and n on behalf of RCVSWL to determine pre- - zoonoses. Journals were perceived as the sent and future service issues by examining a : most important source for 'keeping up-toA full version of this reation he originat at http//wwiwtwales.reeserve.co.uk/WALES/ 0 the use of a computer, hardly any used it for Infosurvey 99 for_web.pdf $\quad$, database searching. 
The same authors' research (1988 [4], : pointed out that such research eventually fil-
ated 1998 with Wiese [5]) into the library updated 1998 with Wiese [5]) into the library : ters down into the practice of tomorrow, bu nary medical a cross-section of US veteri- : missed the fact that his respondents were section on student use of electronic : mation (SDI) service in all but name resources. VETCD, MEDLINE and BEASTCD : In Gerrard's research on veterinary prac-
were the most popular databases used, illuswere the most popular databases used, illus- : titioners' views on the Internet, it was reporttrating the general shift from print to com- : ed that, although the level of Internet general, American veterinary students : awareness was high, most vets were unsure showed themselves to be pragmatic elec- - abfer them and thand the benefits it could tronic resource users, acknowledging that - primary reason for vets not using it [9]. Of his other resources such as colleagues, CPD : sample $46 \%$ had no interest in CPD course courses/workshops and conferences would : aimed at increasing Internet awares continue to be their main sources of infor- : key issue, the author reckoned, if the Interne

The few studies of UK vets that have been undertaken tend towards the enumeration : and quantification of veterinary information journals subscribed to, etc., as opposed to a deeper investigation of IN, IU or ISB. However, 1993 [7]) survey is a sood referen fieldwork findings on computer use in practices. for its use was made of computers for storage or retrieval of veterinary information - accounting and administrative tasks predominated. There was a general lack of awareness of
electronic resources with only $10 \%$ of responelectronic resources with only $10 \%$ of responsources. One third of respondic information happy with the amount of information available and the means of obtaining it. The remaining two thirds expressed some degree of dissatisfaction, being time-pressed vets
who saw themselves faced with a mountain who saw themselves faced with a mountain by VIS ensuring quick and easy access to information.

Raw had come to a similar conclusion some years previously [8]. He drew on a larger sample (537 practices) and chose to focus :
on practice library issues - subscriptions, on practice library issues - subscriptions,
book selection criteria, budgets etc. Respondents accused publishers of selling : out-of-date books (due to the long lead times involved in preparing new editions) and pre- : ferred specialist material aimed at vets in al. found geographic isolation, cost and inad-
equate technology to be the principal barriers practice rather than specialist 'esoteric'
research material appearing in journals. Raw to information use cited by rural health care Various medical ISB/IU studies of practitioners exist. Gravois et al. studied dental hygienperceived barriers to information use as end examined IN/SB/IU of Texas physicians with particular attention to the availability of MEDLINE [12]. The authors found that personal

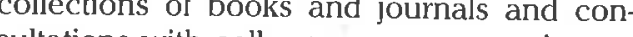
? and would invariably save the time and effort worthy research areas [11]. Bowden et al. to, General Practitioners (GPs)) [13]. Dee and : Blazek reported that, for rural physicians, communication with colleagues provided : required to consult books and journals, even those readily available in the GP's personal library [14]. Work by D'Alessandro et al. on Digital Health Science Libraries for rural physi-
cians may also have implications for veterinary information provision 115 . Finally,
Thompson provides a useful review of the literature on the characteristics of information : resources preferred by primary care physicians with reference to ISB/IU [16]. Outside
of the medical domain, work by Ellis in academic and industrial environments provides a solid theoretical base for user study research on IN and ISB [17, 18, 19, 20].

\section{Methodology}

Questionnaires (paper and email versions) followed-up by semi-structured interviews were the methods of data collection selecta large geographic 'reach', comparably quick and cheap data collection and the opportunity to explore attitudes and opinions in more detail. The main disadvantage is the reliance on the goodwill of time-pressed and '

Topics covered in the questionnaires and interviews were based on key issues identified in the literature review. A pilot sample of vets tested question design, survey layout and closed question responses. The RCVS : 'immediate' nature of email, $i$.e. message

sampling frame, from which a simple random 'it was decided to use Vaper questionnaire, $(n=100)$ as a basis but with compenstion or a longer survey $(\mathrm{n}=149)$. The email ques tonnaire sample $(n=60)$ comprised vets whose email addresses had already been . inform publicity, stamped addressed envelopes, offcial invitations, follow-up letters and a prize draw incentive (as recommended by Opere all used to boost the The interview

The literview stage was conducted after returned. Due to cost, time and field been constraints, seven interviews scheduled to Cast no more than an hour were undertaken. Candidates for interview were selected from . of practice type, IT use, or geographical loca-

\section{Results}

The final response rate was $39 \%(n=82)$. This .

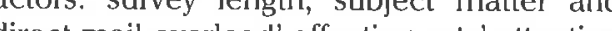
and time The email survey gained a slitity se rate (42\%) than the paper surey (38\%). This may be because of the more can be replied to quickly and easily with a

Table 1. Survey returns and interviews by practice type compared to UK totals

\begin{tabular}{|l|c|c|c|c|}
\hline Practice type & Number (n) & Survey \% & UK \%* & Interviewed \\
\hline Mixed & 28 & 34 & 54 & 1 \\
\hline Large & 5 & 6 & 1 & 1 \\
\hline Equine $(100 \%)$ & 3 & 4 & 3 & 1 \\
\hline Small & 45 & 55 & 37 & 4 \\
\hline Poultry $(100 \%)$ & 1 & 1 & 0.3 & 0 \\
\hline Other & 0 & 0 & 5 & 0 \\
\hline Total & $\mathbf{8 2}$ & $\mathbf{1 0 0}$ & $\mathbf{1 0 0}$ & $\mathbf{7}$ \\
\hline
\end{tabular}

*Source: RCVS Practice Database

Aslib Proceedings Vol 52, No.7, July/August $2000-237$ sample population was thus 209. Prestora 
click of a button (or, indeed, deleted!). Table
1 shows the final split of survey returns and 1 shows the final split of survey returns and mated UK totals.

\section{Computer usage}

All respondents used a computer (rarely an Apple or Unix system). Laptop use was palmtop use $8 \%$. The most popular computer activity was word-processing followed by accounts/invoicing and label printing (in that order). The least popular activity was website design or maintenance followed by veterinary : note taking and database searching. If, for the purposes of analysis, email communication, nary note taking and multimedia use are cersidered to be the principal examples of IS from the list of activities given, the percent-
age of ISB activity out of total computer activity can be calculated. Thus ISB comprised jus puter activity. There was no spondent comence found between paterignificant differactivity by practice type, size, or age group.

\section{The Interne}

The biggest waste of time known to man' lamented one respondent. But the majority of work related purposes and internet for were using it for non-work related purposes. Of the remainder, there was a small group vets who were 'Internet converts', i.e. they stated they would use it if they had the time. Only three respondents had no idea what use the Internet would be to their work. Despite tively high cost of using the on the comparacompared to the USA, due to lneal charges, such costs were not perceived as a barrier to Internet use with only one respondent mentioning them. The remaining $11 \%$ of for nondents had other undeclared reason over half the mixed animal at present. Just dents (54\%) used the Internet for any purpose, as did $69 \%$ of the small animal practice respondents. All the equine practice respon- : dents in the survey used the Internet and all

bar one of the large animal practice respon-

An analysis of Internet use by age reveals were least likely to vet respondents who even), however this category is under represented in the survey. With this in mind, was found that the 31-40 age group were the category using the Net the most (73\%), fol(51-59 by the $41-50$ age group (67\%) and the How did the $(60 \%)$.

ternet resources? Threets find out abou emerge (in rank order, most popular first): online sources (comprising surfing, browsin lype activities, search engines, newsgroups (e) (e.g. adverts, articles in journals or newspaof mouth).

And once these vets are online, wha hen? Some degree of caution should be whercised here as some of the categories of online esource provided were interpreted differentcaspondents. However, it is fair the Internet for work went to pofe used institution websites at least occas possiona $79 \%$ to velerinary information websites. As for the categories of websites that were not visited, $78 \%$ of Internet respondents never used library catalogues on the Web. as null respenses arhaps too strong here ticking a 'never' box is obvied. somebody stronger statement than the person who leaves it blank, so 'do not use' is a more appropriate interpretation. But, semantics aside, the inescapable conclusion is that library catalogues are not being used, in spite of major However, mailing lists are used deve (85\% of Internet respondents did not use (hem) and $74 \%$ of Internet respondents never used newsgroups, publishers' websites, or The low reported in their veterinary work. also important as it shows the databases is : equivalent is needed of the varia a veterinary this was the most cited example MEDLINE

Aslib Proceedings Vol 52, No.7, July/August 2000 - 238 database in the survey - but it is first and foremost intended for the medical profession and so its coverage is not specialised enough for veterinary users. Other frequently cited : Agriculture, Farming and Fisheries (MAFF) site and the BSAVA web pages.

Although fully aware of the Net's poten : tial as an information resource it is fair to say that all the interviewees were at least frustrated by it in its current state, if not openly Net, thanks to its speed and immediacy, as the profession's preferred internal publishing/communication medium for position statements and professional issues. Speed of publication was also a key factor in another's appreciation of FEMS online as immediacy was also valued by a third interviewee as it means there is the third interpossibility of being able to salify any infer-" mation need instantly.

Email

Around $79 \%$ of respondents had the use of an email for work related majority of them used of the survey's vets can be described as the :" 'wired', using both Internet and email to help them with their practice work. An addition $16 \%$ used email and not the Internet in their work and $17 \%$ vice versa.

The majorty of respondents seemed to : were corresponding winthe UK and $69 \%$ other countries. On the other hand $91 \%$ of vets using email for their work did not exchange information with laboratories, Librarlans did not fare well either with only $18 \%$ of he respective sample emailing them. As for just under a third of respond, changing information with these catere expeople. $62 \%$ of respondents who used email for work were communicating with between one and three types of recipient. 9\% could be described as 'email addicts' as they were

the sene 'er more types as recipient. ed information which predominated and case related information appeared to be the most popular type of information bein exanged, closely followed by practice management information. The relatively low -

Although, on the basis of the survey find ings, it can be argued that email use is in its wancy in terms of ISB, some of the interviewees indicated that this situation might  arly and becomes once it is used fairly regu- Another vet, a heavy opinion that email use saved paper on the thing an email address was a vital part of explaining one of the fasciscinners to ew findings - the use of flatbed vet participated in a document images. One which meeting proceedings and journity in cles were exchanged via email and post Another exchanged non-clinical information .

\section{CD-ROMs}

Of the 80 respondents who answered the $12.5 \%$ indicated that they did pot use CDROMs at all, while the majority $(40 \%)$ had not used a CD-ROM in the previous month (19 of (hese were Internet users, 13 were not). The CD-ROM users as only 3 were hardly heavy CD-ROMs 15 or more times in the preved CD-ROMs were used mainly for 'staying currenl' (presumably as part of the vets' contimuing professional development requirepares with 55\% of regular users. This comCD-ROMs to answer patient are consulting and $36 \%$ consulting them for persons ses. It was also found that regular CD-ROM users overwhelmingly $(89 \%)$ preferred to search CD-ROMs by themselves . 
Vetstream products dominate the veterinary CD-ROM market. Twenty respondents
reported using CD-Canis, while twelve used reported using CD-Canis, while twelve used
the CD-Felis and five CD-Equus. Eight respondents used Vision. The response for MEDLINE (3) was probably not accurate as many respondents may have considered it to me any Internet resource (e.g. via Grateful Med) rather than a CD-ROM or database.

Vetstream CD-ROMs were discussed in thee interviews. Opinions were negative on to be expensive, clumsy to navigate through and conservative in that they do not $\mathrm{gos}$ beyond the standard techniques that most vets know anyway. One vet obviously appreciated CD-Canis and CD-Felis as he worked essentially alone in a small animal practice included on them obviated an online verion He did, however, acknowledge their limited scope/coverage. Another vet had looked CD-Equus and found it to be expensive and once again, not particularly detailed conpared with journal articles. Another found the available on Finally, one interviewee signalled an information need fulfilled particularly well by the CD-ROM medium - the communication of conference proceeding

Are CD-ROMs threatened by the Internet? Although the third interviewee was proshe found that she wasted a lo databases on the Internet. The fifth saw no need to tie up his telephone line when he had : perfectly adequate information on CD-ROM. The sixth had suffered slow download time when online which made him appreciate CDwell as the functionality of and portability as He was also concerned about general image
quality. The fourth vet thought that CD-ROMs would replace paper journals, something that would facilitate indexing and searching. The first interviewee, however, was not impressed
with the CD-ROMs she had seen due to their basic level. For her, books provided a viable alternative to the Internet.

The survey revealed four major reasons

for CD-ROM non-use: lack of easy access, lack of time, lack of equipment and uncertainty wer which databases to use. Reasons which equally lowidered important (and scored wrong kind of information and no access ournals once references found.

Preferred communication media The closed question relating to this issue did not generate an entirely satisfactory set of multiple answers when only ondents gave was required. However, the rank order of ferred media is unchanged after using either an elimination or compensation strategy in analysis: (favourite first) email, paper document in the post, Internet site, fax, telephone. that no distinction was masselte/other. Note ent kinds of telephone Fax and telephore almost interchangeable in the rankings. For the 18 respondents who stated that neither they nor their practice had an email address information in paper document form through by fost was the prefered medium followed Respondents' prefer

ing information when working away of obtainpractice was also explored in order to gauge the potential use of portable communication/information devices. Out of the 61 stated thents who answered the question, 5 the practice to get the informey retumed to ed. Fifteen used mobile telephones An additional 31 vets said they used the telephone, which may or may not include mobile equipment. Interestingly, 7 vets stated they used books. Laptop use was reported by 8 respon-

Preferred information sources However many respondents might be using

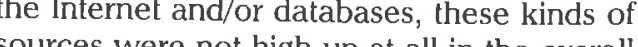
scheme of veterinary information seeking ferences wornal articles, textbooks and con- diagnostic and therapeutic information. Use : information sources for each information of other sources varied. It was found, for : type, while the equivalent figure for small aniexample, that promotional literature was con- : mal practices was six. Once again, these figsidered to be a particularly important source : used it), as werion ( $77 \%$ of respondents : used it), as were company representatives other hand, the relatively low overall wise of databases and websites is probably a reflection of the quality of information provided and, especially in the case of websites, of a : lack of crediblity the. siles that we biased and : are neither peer-reviewed nor officially ures should be treated with caution because For size and response rate.

For drug information needs, it can be argued that respondents working in small as an information source to a much gercer extent than their peers in mixed animal prac ces (76\% versus $32 \%)$. A similar relationship " (n) aso using animal practice respondents were a greater extent $(+17 \%$ in the casources to Information source use by practice type A tentative analysis was made of variations in source use for each information type by small A : encyclopaedia and compendia than smal

\begin{tabular}{|l|r|r|r|r|r|r|r|r|}
\hline Information source* & $\begin{array}{c}\text { Drug } \\
\text { (n) }\end{array}$ & Rank & $\begin{array}{c}\text { Diag } \\
\text { (n) }\end{array}$ & Rank & $\begin{array}{c}\text { Ther } \\
\text { (n) }\end{array}$ & Rank & $\begin{array}{c}\text { Total } \\
\text { (n) }\end{array}$ & Rank \\
\hline Journal articles & 72 & 1 & 78 & 1 & 73 & 1 & 223 & 1 \\
\hline Textbooks & 71 & 2 & 71 & 2 & 73 & 2 & 215 & 2 \\
\hline Conferences & 63 & 3 & 71 & 2 & 69 & 3 & 203 & 3 \\
\hline Promotional literature & 63 & 3 & 29 & 12 & 47 & 8 & 139 & 8 \\
\hline Company representatives & 60 & 5 & 18 & 18 & 40 & 10 & 118 & 10 \\
\hline Practice colleagues & 56 & 6 & 64 & 5 & 63 & 4 & 183 & 4 \\
\hline Training courses/workshops & 48 & 7 & 61 & 6 & 57 & 6 & 166 & 6 \\
\hline Conference proceedings & 47 & 8 & 51 & 7 & 50 & 7 & 148 & 7 \\
\hline Encyclopaedia/compendia & 45 & 9 & 31 & 11 & 38 & 11 & 114 & 11 \\
\hline Other vets & 45 & 9 & 65 & 4 & 61 & 5 & 171 & 5 \\
\hline Personal notes/files & 43 & 11 & 43 & 8 & 42 & 9 & 128 & 9 \\
\hline Current awareness publications & 31 & 12 & 35 & 10 & 33 & 12 & 99 & 12 \\
\hline Practice records & 30 & 13 & 25 & 13 & 29 & 13 & 84 & 13 \\
\hline Other books & 25 & 14 & 23 & 14 & 29 & 13 & 77 & 14 \\
\hline Databases & 21 & 15 & 22 & 15 & 21 & 15 & 64 & 16 \\
\hline Internel sites & 20 & 16 & 21 & 17 & 21 & 15 & 62 & 17 \\
\hline References/citations & 18 & 17 & 22 & 15 & 18 & 17 & 58 & 18 \\
\hline Abstracting/indexing services & 17 & 18 & 17 & 19 & 15 & 19 & 49 & 19 \\
\hline Laboratory & 15 & 19 & 42 & 9 & 17 & 18 & 74 & 15 \\
\hline Annual reviews & 10 & 20 & 10 & 20 & 11 & 20 & 31 & 20 \\
\hline Other & 7 & 21 & 5 & 21 & 5 & 21 & 17 & 21 \\
\hline
\end{tabular}

Respondents could select more than one source

Aslib Proceedings Vol 52, No.7, July/August $2000-241$ 
overall finding that journal articles were the ${ }^{*}$ therapeutic information than the mixed anipreferred resource for drug information still :
holds, textbooks, promotional literature and company representatives were of equal : Information source use by practice size importance to mixed animal practitioners - A couple of observations can be made. Firstly, more than the over

hierarchy' fore defined 'information : sources on the small animal side than on the : mixed animal. In other words, mixed animal practice respondents were making greater use of a wider range of resources for this kind tice peers On reflection this is col pracsense - the wide variety of animal species : seen by mixed animal practices, with all their respective conditions, must make calls on a : wider variety of diagnostic information
sources than is needed for a small animal sources than is needed for a small animal
practice. And it is this variety that may explain the greater use by mixed animal practice respondents of particular information resources such as training courses/workshops $(+11 \%)$, practice colleagues $(+8 \%)$ and laboratories $(+13 \%)$ to help them cope.

Once again, small animal practices made greater use of databases for diagnostic infor(33\%:11\% and $47 \%: 36 \%$ respectively). : However, there was a roughly similar use of Internet sites, albeit low in priority. One curiosity is that the small animal practice
respondents reported significantly greater respondents reported significantly greater
use of promotional literature $(+23 \%)$ for this type of information. As suggested previously, in conjunction with other being used sources.
For therapeutic information, training courses/workshops featured more prominently in mixed animal practice respondents'
routines $(79 \%$ versus $67 \%)$, as did the use of routines $(79 \%$ versus $67 \%)$, as did the use of
practice records ( $46 \%$ versus $31 \%)$. On the other hand, current awareness publications, personal notes/iles, references/citations, databases, abstracting/indexing services and :
laboratories were all used more (at least laboratories were all used more (at least
$+10 \%$ for each one) by small animal practice : respondents. The small animal practices in resources by 'super' (i.e. $11+$ vets) practices especially use of databases. This practice type the survey did not use any services or annua . ed very low use of references/citations.
Further research is clearly needed here confirm or deny these findings.

For one thactices a clustering effect - can be observed especially for diagnostic and " herapeutic information. That is, a small group of sources were used consistently more than . inces. Only vets and conference proceedneeded were additional sournation was - similar extent: promotional literature, comAs for small (1-3 man) practices, it is fair to say that they generally used a wider variety of thes than or size practices.

- useful information sur high - the mos information along with textbooks and second most useful source for diagnostic information . is expected that this trend would also apply -

\section{Keeping up-to-date}

Journals appear to be the most popula (Table 3). However, it can be argued that " ' 'many to one' oral information source and the most used Certainly, of source that is 'al sources. Responses such as 'CPD' and 'eading' conceivably included both journa (n) books and courses and so cloud the electronic information sources (and possibly one
Table 3. Answers to open question - how do you keep up-to-date?

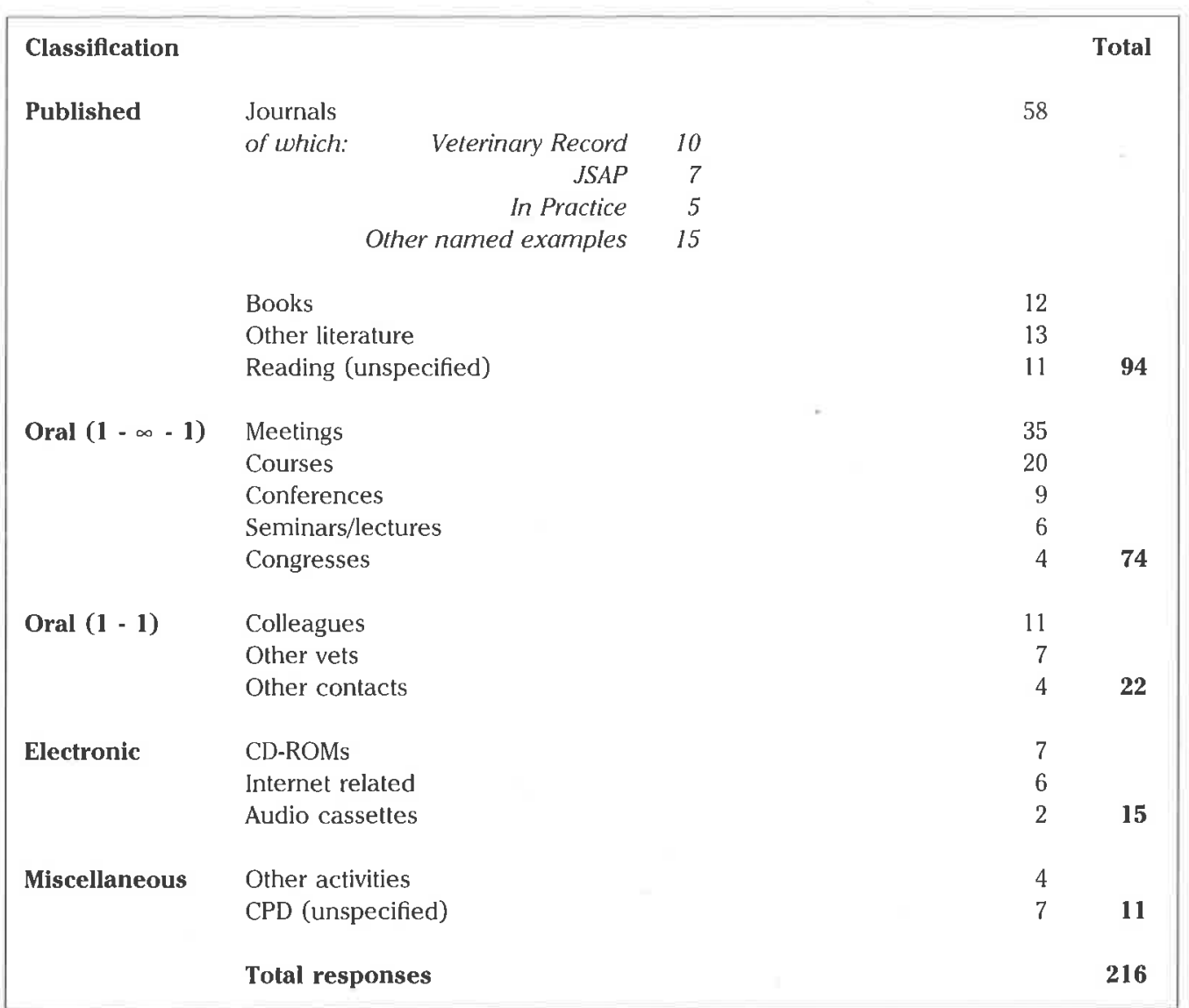

being important, despite the fact they both $:$ Barriers to IU

offer the possibility of being the most current ${ }^{*}$ Time, cost and inadequate information skills

training (in that order) were regarded as the main barriers to IU. Time barriers were twice A similar conclusion was reached for respon- $\quad:$ as significant for respondents as cost barriers. dents' optimum sources of information in : tional question gave respondents the oppor(textbook or reference) was the main life sup- " the time problem extracted from previous port system (sic) with $45 \%$ of responses fol- $:$ research. The results showed a very marginlowed by practice colleagues with $18 \%$. There - al preference for the 'circuit librarian' idea is really little to choose between the remain- : (+2) over "onsite information intermediary' ing responses. Electronic indomation sources : and personal hitermedlary at informaterwere clearly not regarded as being sourt

However, the largest response was comprised of don't knows, no preferences and 
null responses $(48 \%)$. This can be interpreted in several ways. Firstly, as a genuine expres-
sion of ignorance. Secondly, as a passive sion of ignorance. Secondly, as a passive
response, e.g. it is not for me to know the response, e.g. it is not for me to know the Thirdly, as an acknowledgement that there : may not be a definite answer, with it being left to the individual vel's discretion to decide what action to take (if any) about time pres-
sures. It should also be noted that respondents were given space to suggest their ow

\section{Problems with information sources} 4) was the most pressing problem with ve having to grapple with out-of-date information. One respondent pointed out that rapid : cially in disciplines such as anaesthesia, are rendering textbooks out-of-date as soon as
they are published. Problems with availability and content here are also of concern. The former category encompasses occasions
where colleagues or referral vets are unavailwhere colleagues or referral vets are unavail-
able at the time when the information need able at the time when the information need : at home or the information is at another pracice in whatever form Content problems refer : to poor coverage of a topic, lack of detail on : rarer topics, or conflicting/biased advice.

\section{Table 4. ISB problems}

\begin{tabular}{|l|l|c|}
\hline Problem & Example(s) & Number $(\mathbf{n})^{*}$ \\
\hline Currency related & Out-of-date & 19 \\
\hline Availability & Colleague busy & 16 \\
\hline Content related & Biased, irrelevant & 16 \\
\hline Physical & Bulky, hard to file & 9 \\
\hline Design related & Poor indexing & 9 \\
\hline Time consuming & - & 8 \\
\hline Speed of access/delivery & Internet downloads & 8 \\
\hline Finding the known reference & - & 4 \\
\hline Lack of resource awareness & Where to look? & 3 \\
\hline Too many sources & - & 3 \\
\hline Other & Cost & 3 \\
\hline Total responses & & $\mathbf{9 8}$ \\
\hline
\end{tabular}

*Respondents could specify more than one problem.
The fact that the cost of information does ngine but also for paper jounals. Finally, at the risk of being provocative major problem was respon-

\section{Evaluation of information}

'Credibility of source' was by far the most sigof 76 respondents allocating it first or second experience' came second $(n=38)$ and 'discussion with practice colleagues' in third place $(n=25)$. 'Methodology used' and 'conhardly used as evaluating criteria.

\section{eterinary libraries}

W of respondents used a veterinary library 政 who did not use veterinary libraries, 21 were . cause and effect. The opportunity was taken o ask these 37 non-users what (if anything) would prompt them to use a veterinary library position out of the list provided 'Persona

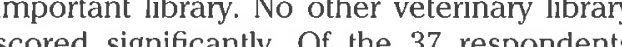

and the majority of responses stated 'improve : vets in this survey were using the Net for their access via the Internet', i.e. accessing library * work than were in Gerrard's survey (68\% verresources online to downibad entire anticles, : sus $28 \%$ ) and there appeared to be less uncermajority of library users Veterinary libraries were primarily used * using the Net for their work than reported for article/reference chasing ( $43 \%$ of the $69{ }^{*}$ using a veterinary library (by whatever responses to the question). The more gener- : means). This is in spite of the fact that al literature searching activity accounted for a : RCVSWL and, recently, the Royal Veterinary further $19 \%$ of responses. Book or journal : College, have both invested in Sirsi's UNI-

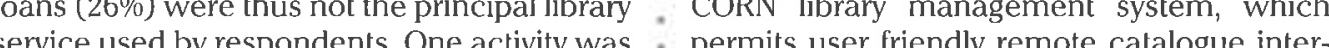
mentioned that had not been previously con- * rogation/interaction via the client World Wide sidered - a minority of respondents were using veterinary libraries to evaluate textbooks before purchasing them.

\section{A private affair?}

A an interviewee that any ISB should be don in private, that is to say, not in front of the client. This arose when discussing the potential use of portable data assistants when on
call - the vet was adamant that consulting any call - the vet was adamant that consulting any as it was not seen to be 'professional'.

\section{Conclusions}

At first, it seems that the findings from this research merely confirm those published prewously. Journals and lexbooks conlinue to :" litioners in this survey's sample, just as they : were some 20 years ago when Drake and Woods conducted their research. Despite : advances in technology, books are still the emergency information source par excellence as (re)confirmed by Pelzer and Leysen in 1991. Even if more practices in this survey had Valleley in $1993(77 \%)$, the same conclusion applies now as did then - administrative tasks : such as word-processing and accounting comprise the majority of computer activity, : despite the arrival of the Net and email. What is more, the major barrier to IU identified by Raw

New ground has been covered, though, especially with regard to the Internet. More
Web browse

Survey comments and interviews revealed that lack of publicity was partly to blame, but mainly it was the assumption that such systems fulfiled a proven practitioner Internet In reality, it was found that there was a far stronger demand by VIS users and nonusers alike for online full-text journal information and that an opportunity existed for the assume a leading Internestion's library', to useful from the useless on be lif of the pro- fession. The principal of enhanced online veterinary information provision is thus the key service implication for RCVSWL that can be Thed from current veterinary ISB/IU. This should not be a surprise. It was

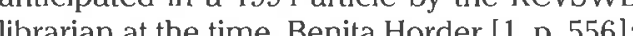
Future possibilities for our users may well include dial-up access, not only to our catalogue, but also CD-ROM databases. Electronic publishing is likely to tinuing professional develop for conlead to increasing use Individual access to databases is likely to aidespread, but there will still be a need for the librarian as intermediary and to provide the

Horder was writing just before the advocating direct dial-up as opposed to dialAslib Proceedings Vol 52, No.7, July/August $2000-245$ speciase over the next decade. Further 
However, her predictions then are now a real- : five Texas counties. Bulletin of the Medical

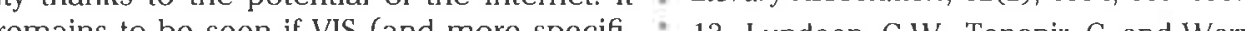

cally, RCVSWL) are willing and able to tap it : P. Information needs of rural health care practi-

\section{References}

1. Horder, B. A profession's library, Veterinary Record, 134(21), 1994, 554-556.

2. Pelzer, N.L. and Leysen, J.M. Use of informa- : Bulletin of the Medical vibrany Association $79(1)$ Bulletin of the

3. Drake, M.A. and Woods, L.A. An information : service for practising veterinarians. Bulletin of the Medical Library Association, 66(4), 1978, 4. Pelzer, N.L. and Leysen, J.M. Library use and A Association, 76(4), 1988, 328-333

5. Pelzer, N.L., Wiese, W.H. and Leysen, J.M.
Library use and information-seeking behaviour

Library use and information-seeking behaviour
of veterinary medical students revisited in the of veterinary medical students revisited in
electronic environment. Bulletin of the Medice
Library Association, 86(3), 1998, 346-353.

6. Bawden, D. and Valleley, C. Veterinary information: sources and us

7. Valleley, C. The provision of information for Scc in If ormation Science dissertation University, 1993.

8. Raw, M.E. Survey of libraries in veterinary
practice. Veterinary Record, $121(6), 1987$

9. Gerrard, B. Vets on the net (3): present and 602-606.

0. Biggs, $\mathrm{H}$ IT and the vet. Produce Studie Research. [Cited in: News review, Veterinar limes, August 1999, 36.

11. Gravois, S.L., Fisher, W., Patrick, S.C. and Bowen, D. Information-seeking practices of den-
tal hygienists. Bulletin of the Medical Library Association, 83(4), 1995, 446-452.

12. Bowden, V.M., Kromer M.E. and Tobia, R.C. tioners iation needs of rural health care practiAssociation, 82(2), 1994, 197-205.

14. Dee, C. and Blazek, R. Information needs of (4) 259-264.

15. D'Alessandro, D.M., D'Alessandro, M.P.,
Galvin, J.R, Kash, J.B, Wakefield D.S. and Erkonen, W.E. Barriers to rural physician use of a digital health sciences library. Bulletin of the
Medical Library Association $86(4)$ 583-593.

16. Thompson, M.L. Characteristics of inform cians. Bulletin of the Medical Library Association,

17. Ellis, D., Cox, D. and Hall, K. A comparison the information seeking patterns of Journal of Documentation, $49(4), 1993,487-501$.
Jose 18. Ellis, D. Modelling the information-seeking pateons of academic researchers: a grounded $469-486$

19. Ellis, D. and Haugan, M. Modelling the information-seeking patterns of engineers and research scientists in an industrial environment.
Journal of Documentation, 53(4), 1997, 384-403. 20. Wilson, T.D. Information behaviour: an interdisciplinary perspective. Information Processing 21. Vet Web, http://www vetweb.

22. Vet Net. http://www.vetnet.co.uk 23. Vetindex hitp:/www vetindex couk

24. Oppenheim, A.N. Questionnaire design, interviewing and attitude
London: Pinter Publishers, 1992.

The following people are thanked for their mualuable assistance; Liz Armitage, David Jackson, Washiela Jacobs, Simon Jackson, Yioda Panayiotou, Brian Wales and Tom Roper.

\section{The century of film: bibliographic control and legal deposit of the moving image}

Stephen Pearson

British Film Peditute National Library, Stephen Street, London WIP 2LN stephen.pearson@bfi.org.uk

1895 saw the dawn of film - a new publication medium that we associate particularly with the century that has just ended. The survival of films and of information about ther has been severely hampered in the UK by the abseste but might for the new digital media help to prevent the loss of treasures on the scale of that suffered by film in the twentieth century.

Introduction

Kinematograph Weekly and its various relat
ed titles. Of the films from the 'sound' era new century to look back at the state of our knowledge about the publications of the pre-
vious one. It seems particularly appropriate vious one. ll seens part culany appopiate : medium belongs almost entirely to the previous century - indeed it represented a revolutionary technological innovation at the start of : the twentieth century. Such a review might help us to learn some lessons, and this is very
appropriate at a time when methods of proappropriate at a time when methods of proare underging dramatic changes, because of the advent of new technologies. Of the films that were produced in this country during the first thirty years of the existence of film technology, in other words the 'silent' era, probably no more than $20 \%$ survives. We have to use he word probably : of all the films that were produced and we shall probably never be absolutely sure what has been lost. Even now occasional "treasures' are rediscovered. In the absence of any : formal requirement to register films, such as that established by the Library or Congress in : the United States, the Linfornaton that we do has had to be painstakingly pieced together : from surviving copies of company film catalogues, from the surviving films and fragments of films, and from the trade magazines of the ed titles. Of the films from the 'sound' era, are rather better because, following the Cinematograph Films Act of 1927, it became their footage with the Board of Trade.

\section{The development of film} catalogues

-

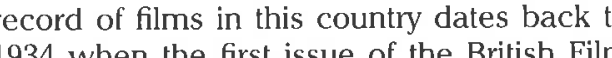
- Institute's Monthly Film Bulletin appeared. Al that time the film records were very brief: THIS IS THE LIFE. British Lion Censor's Certificate ' $\mathrm{W}$. Broad comedy with Binnie Hale and

The covered educationa established the tradition of including not jus British films but also those imported into this The As the foreword to the first issue said: The British Film Institute, in deciding educational and documentary fims is endeavouring to meet a difficulty which has been experienced for sometime past by various organisations and individuals who have realised the possibilities of the film and haps 50\% survives. For this period the record 